\title{
Standardisation of Travel Planners and Use of a Return Channel
}

\author{
Grzegorz Sierpiński, Marcin Staniek, Elżbieta Macioszek \\ Silesian University of Technology, Faculty of Transport, Department of Transport System and Traffic Engineering \\ Krasińskiego 8 Street, 40-019 Katowice, Poland \\ grzegorz.sierpinski@polsl.pl; marcin.staniek@polsl.pl; elzbieta.macioszek@polsl.pl
}

\section{Extended Abstract}

\section{Introduction and Scope}

For the sake of making decisions on changes in urban transport systems, one requires knowledge of transport-related needs of the travelling population. Only appropriate identification of locations where travels are generated and absorbed as well as travel-related motivations enables a coherent system fully integrated with the city to be created. Contemporary technologies allow for rapid data exchange, increasingly often making it possible to transfer information in transport systems as well (e.g. dynamic notifications in public transport stops, motion detectors, information on availability of parking places, car sharing portals etc.). The information sharing applications include travel planners ([1] and [2]). What they provide for is recommending an optimum way of travelling to the user in accordance with pre-defined criteria and with reference to the chosen travel mode. The percentage of persons using this specific source of transport system related information has been regularly growing. Both this phenomenon as well as the fact that one can observe an increasing number of customised travel planners emerging in the market provide grounds for seeking a universal solution based on the maximum available number of the Open Access type data sources. On the other hand, it may be presumed that, in the nearest future, along with an intense growth in the number of travel planner users in the given area, they may be considered as a sample reflecting certain features of travel behaviours observed in the population.

\section{Objectives and Results}

The research objective was to develop a travel planner which, on the one hand, could offer as many options of route and travelling mode searching as possible, and on the other hand, would support local authorities in making decisions concerning further development of the municipal transport system. Green Travelling Planner (GT Planner) [3] is a tool developed as one of deliverables of the international project entitled "A platform to analyze and foster the use of Green Travelling options" implemented under the ERA-NET Transport III Future Travelling programme and co-financed by the National Centre for Research and Development. First result is GT Planner which enables route searching according to four criteria (which account for time, distance and travel expenses, but also environmental impact). At the same time, it offers a choice of one among 11 travelling modes (including e.g. electric cars, transport chains using Park\&Ride and Bike\&Ride systems, travelling by means of urban bicycle or car rental services as well as conventional modes of passenger car, public transport, bicycle, walking etc.). Such a wide array of available options makes it possible, on the other hand, to gain knowledge about the choices made by the travelling population. The queries submitted by users are archived by means of the return channel [4]. Owing to this approach, it is possible to learn not only about the transfers actually completed on a daily basis, but more importantly, about the actual needs. As a research result, beside the tool, procedure to support a decision making problem was develop. This four-step procedure allows to introduce changes according to real needs in the scope of organisation (e.g. by altering routes of bus lines, modifying timetables, introducing parking fees and charges for entering the given city district) as well as transport infrastructure (e.g. implementing a system of bicycle or electric urban car rental services, building dedicated bus lanes).

\section{References}

[1] P. Borkowski, "Towards an Optimal Multimodal Travel Planner-Lessons from the European Experience," Advances in Intelligent Systems and Computing, vol. 505, pp. 163-174, 2017. 
[2] D. Esztergár-Kiss and Cs. Csiszár, "Evaluation of multimodal journey planners and definition of service levels," International Journal of Intelligent Transportation Systems Research, vol. 13, pp. 154-165, 2015.

[3] G. Sierpiński, "Technologically advanced and responsible travel planning assisted by GT Planner," Lecture Notes in Network and Systems, vol. 2, pp. 65-77, 2017.

[4] G. Sierpiński, E. Macioszek, M. Staniek, I. Celiński, "Big Data Concerning Travel Preferences as a Means to Support Decision Making in the Field of Environmentally Friendly Urban Development," in New Pressures on Cities and Regions Conference Proceedings of the Regional Studies Association Winter Conference, London, pp. 8485, 2016. 